\title{
Acute encephalopathy with biphasic seizures and late reduced diffusion
}

INSERM

\section{Source}

INSERM. (1999). Orphanet: an online rare disease and orphan drug data base. Acute encephalopathy with biphasic seizures and late reduced diffusion. ORPHA:363549

Acute encephalopathy with biphasic seizures and late reduced diffusion (AESD) is a rare childhood-onset epilepsy syndrome associated with infection and characterized by a biphasic clinical course. The initial symptom is a prolonged febrile seizure on day 1 (the first phase). Afterwards, patients have variable levels of consciousness from normal to coma. Irrespective of the consciousness levels, magnetic resonance imaging (MRI) during the first 2 days shows no abnormality. During the second phase (usually days 4 6), patients show a cluster of seizures and deterioration of consciousness. Diffusionweighted images (DWI) on MRI reveal the brain lesions with reduced diffusion predominantly in the subcortical white matter. After the second acute phase, consciousness levels improve with the emerging focal neurological signs. Neurological outcomes of AESD vary from normal to mild or severe sequelae including cerebral atrophy, mental retardation, paralysis and epilepsy. 